\title{
PERFIL DAS GESTANTES ADOLESCENTES SEGUNDO FREQUÊNCIA AO PRÉ- NATAL E IDADE GESTACIONAL EM FEIRA DE SANTANA-BA, NO PERÍODO DE 2012-2014
}

\author{
Caroline Andrade Araujo1; Maria Conceição Oliveira Costa ${ }^{2}$; Lorena Ramalho Galvão \\ e Jamilly de Oliveira Musse ${ }^{4}$ \\ 1. Bolsista PIBIC/CNPq, Graduando em Enfermagem, Universidade Estadual de Feira de Santana, e-mail: \\ carolline.andr@gmail.com \\ 2. Orientador, Departamento de Saúde, Universidade Estadual de Feira de Santana, e-mail: oliveiramco69@gmail.com \\ 3. Participante do núcleo de Estudos e Pesquisas na Infância e Adolescência, Mestranda do Departamento de Saúde, \\ Universidade Estadual de Feira de Santana, e-mail: lore.galvão@ hotmail.com \\ 4. Participante do núcleo de Estudos e Pesquisas na Infância e Adolescência, Professora do Departamento de Saúde, \\ Universidade Estadual de Feira de Santana, e-mail: musse_jo@ hotmail.com
}

PALAVRAS-CHAVE: Gravidez na adolescência; Pré-natal; Idade gestacional.

\section{INTRODUÇÃO}

A gravidez na adolescência carrega consigo além de potenciais repercussões, no plano existencial, repercussões no plano biológico-social, de maior concentração de agravos à saúde materna, bem como de complicações perinatais. No mundo, cerca de um quarto das mulheres têm seu primeiro filho antes de completar 20 anos de idade, com taxas ainda mais elevadas em países subdesenvolvidos e em desenvolvimento (GOLDENBERG et al., 2005; IBGE, 2010; MARTINS et al., 2011).

Inúmeros aspectos caracterizam a gestação nesta fase como risco, como o difícil acesso aos serviços de saúde, o baixo nível socioeconômico, comportamentos de risco, hábitos e nutrição inadequada, demonstrando a necessidade de controle dos fatores associados à evolução e ao desfecho da gestação e condições de saúde materno-infantil (SANTOS et al., 2014).

Este estudo teve como objetivo caracterizar o perfil das gestantes adolescentes segundo a frequência ao pré-natal e à idade gestacional no momento do parto, como objetivos específicos analisar as características sociodemográficas, do pré-natal e de nascidos vivos de mães adolescentes e avaliar a possível associação entre a gravidez de adolescentes e a ocorrência de nascidos vivos prematuros.

Considerando que a maternidade precoce é um tema de grande relevância, é importante o desenvolvimento de estratégia para intensificar as ações educativas, em particular, sobre a sexualidade e saúde reprodutiva. Os profissionais de saúde devem se qualificar para oferecer à atenção de qualidade no pré-natal desenvolvendo estratégias de incentivo para as mães adolescentes darem continuidade ao acompanhamento.

\section{METODOLOGIA}

Trata-se de um estudo transversal, com base em dados secundários, do Sistema de Informação sobre Nascidos Vivos (SINASC), obtidos através do Departamento de Informática do SUS (DATASUS), disponibilizados pela $2^{\text {a }}$ Diretoria Regional do Estado da Bahia (DIRES) do município. A população do estudo compreende mães adolescentes 10-14 anos e 15-19 anos segundo classificação da Organização Mundial da Saúde/OMS, cujos partos ocorreram em Feira de Santana, no período 2012 a 2014.

As variáveis do estudo foram classificadas em sociodemográficas (faixa etária materna, estado civil, escolaridade); relacionadas à gestação e parto (número de consultas pré- 
natal, tipo de parto - normal ou cesariano); relacionadas ao RN (peso de nascimento e idade gestacional). Os RN foram classificados segundo o Ministério da Saúde (MS) em de baixo peso $(<2500 \mathrm{~g})$; de peso insuficiente $(2500-2999 \mathrm{~g})$ e peso adequado $(\geq 3000 \mathrm{~g})$; prematuros $(<$ 37 semanas). Quanto ao pré-natal este foi classificado em incompleto ( $<6$ consultas) e completo (> 6 consultas) (BRASIL, 2005).

Os dados foram analisados através do software estatístico Statistical Package for Social Science (SPSS) 17.0 e tiveram seus resultados expressados em razão de odds (OR) com intervalo de confiança $(95 \%)$.

\section{RESULTADOS E/OU DISCUSSÃO}

O total de registros de nascidos vivos de mães adolescentes foi de 4820, sendo o percentual de nascidos vivos de mães entre 10-14 anos menor que entre as mães de 15-19 anos, ainda assim é um fato preocupante porque a gravidez precoce pode trazer graves complicações tanto para mãe quanto para criança. Alguns aspectos que podem caracterizar a gestação nesta fase como riscos são: baixo nível socioeconômico, reduzido acesso aos serviços de saúde, hábitos e nutrição inadequada e comportamentos de risco, podendo caracterizar a gravidez na adolescência como um fenômeno complexo, necessitando de atenção especial para que haja um desenvolvimento saudável da gestação (SANTOS et al., 2014).

Quanto à escolaridade, a maioria das adolescentes frequentaram a escola, porém $34,5 \%$ das adolescentes tiveram $\leq 7$ anos de estudos e $32,5 \%$ tiveram $\geq 8$ anos de estudos, demonstrando que existe um elevado número de adolescentes que se tornaram mães antes de completar o segundo grau. Pesquisas apontam que o abandono dos estudos é um fator marcante durante a gravidez na adolescência, sendo assim, um risco social. Sobre o estado civil observou-se que a maioria das adolescentes não tinham parceiros, e estudos demonstram que é importante o suporte dado pelo parceiro no acompanhamento da gestação, sendo considerado como fator de proteção para os transtornos ocasionados neste período (RODRIGUES, ALMEIDA, RAMOS; FERNANDES, 2017).

A realização do pré-natal principalmente com início no primeiro trimestre da gestação, tem grande relevância na prevenção de complicações materno-infantil, visto que o objetivo do acompanhamento é assegurar o desenvolvimento da gestação permitindo o parto de um recém-nascido saudável. Foi observado que a maioria das adolescentes não tiveram nenhuma consulta de pré-natal deixando-as expostas às complicações, sem terem suporte para os tratamentos necessários (BRASIL, 2013).

Segundo o MS, é considerado prematuro quando o RN é pré-termo apresentando idade gestacional (IG) inferior a 37 semanas e Baixo Peso ao Nascer (BPN) quando o RN tem peso inferior a 2.500 gramas (BRASIL, 2015). Em 2012, tiveram maior número de RNs < 2.500 gramas e quando o RN nasce de baixo peso pode comprometer o início da amamentação, podendo afetar o vínculo materno-infantil devido a introdução de suporte nutricional para estabilização da situação de risco (PONTES et al., 2013).

Nos dados relacionados à IG, embora o número de nascidos vivos prematuros apresentarem porcentagem inferior aos nascidos vivos $\geq 37$ semanas, foi observado que houve aumento nos casos de RNs prematuros no período de 2012 a 2014, o que demonstra necessidade de atenção especial no acompanhamento pré-natal visando redução dos riscos para prematuridade (BRASIL, 2013). Em relação ao tipo de parto observou-se que ocorreram maior porcentagem de partos vaginais, podendo estar relacionado ao nível socioeconômico. Segundo Gama (2014), a cesariana esteve mais fortemente associada às melhores condições de vida das adolescentes por possuírem plano de saúde. 
Tabela 1. Características sociodemográfica, de pré-natal e de nascidos vivos de mães adolescentes, SINASC, 2012-2014. Feira de Santana - BA.

\begin{tabular}{|c|c|c|c|c|c|c|c|c|}
\hline & \multicolumn{2}{|c|}{2012} & \multicolumn{2}{|c|}{2013} & \multicolumn{2}{|c|}{2014} & \multicolumn{2}{|c|}{ Total } \\
\hline & $\mathrm{N}$ & $\%$ & $\mathrm{~N}$ & $\%$ & $\mathrm{~N}$ & $\%$ & $\mathrm{~N}$ & $\%$ \\
\hline \multicolumn{9}{|l|}{ Idade materna } \\
\hline $10-14$ & 103 & 6,3 & 103 & 6,7 & 98 & 5,9 & 304 & 6,3 \\
\hline $15-19$ & 1532 & 93,7 & 1425 & 93,3 & 1559 & 94,1 & 4516 & 93,7 \\
\hline Total & 1635 & 100 & 1528 & 100 & 1657 & 100 & 4820 & 100 \\
\hline \multicolumn{9}{|l|}{ Escolaridade } \\
\hline Nenhuma & 3 & 0,2 & 2 & 0,1 & 2 & 0,1 & 7 & 0,1 \\
\hline$\leq 7$ anos & 501 & 30,6 & 613 & 40,1 & 547 & 33 & 1661 & 34,5 \\
\hline$\geq 8$ anos & 394 & 24,1 & 506 & 33,1 & 666 & 40,2 & 1566 & 32,5 \\
\hline Ignorado & 737 & 45,1 & 407 & 26,7 & 442 & 26,7 & 1586 & 32,9 \\
\hline Total & 1635 & 100 & 1528 & 100 & 1657 & 100 & 4820 & 100 \\
\hline \multicolumn{9}{|l|}{ Estado civil } \\
\hline Solteira & 1039 & 63,5 & 1205 & 78,9 & 1351 & 81,5 & 3595 & 74,6 \\
\hline Casada/União estável & 144 & 8,8 & 174 & 11,4 & 170 & 10,3 & 488 & 10,1 \\
\hline Viúva/Divorciada & 1 & 0,1 & 5 & 0,3 & 8 & 0,5 & 14 & 0,3 \\
\hline Ignorado & 451 & 27,6 & 144 & 9,4 & 128 & 7,7 & 723 & 15 \\
\hline Total & 1635 & 100 & 1528 & 100 & 1657 & 100 & 4829 & 100 \\
\hline \multicolumn{9}{|l|}{ Idade gestacional } \\
\hline$<37$ semanas & 63 & 3,9 & 87 & 5,7 & 102 & 6,2 & 252 & 5,2 \\
\hline$\geq 37$ semanas & 375 & 22,9 & 699 & 45,7 & 944 & 56,9 & 2018 & 41,9 \\
\hline Ignorado & 1197 & 73,2 & 742 & 48,6 & 611 & 36,9 & 2550 & 52,9 \\
\hline Total & 1635 & 100 & 1528 & 100 & 1657 & 100 & 4820 & 100 \\
\hline \multicolumn{9}{|l|}{ Consulta pré-natal } \\
\hline Nenhuma & 710 & 43,4 & 521 & 34,1 & 841 & 50,8 & 2072 & 43 \\
\hline 1 a 6 consultas & 570 & 34,9 & 578 & 37,8 & 525 & 31,7 & 1673 & 34,7 \\
\hline$>6$ consultas & 283 & 17,3 & 309 & 20,2 & 234 & 14,1 & 826 & 17,1 \\
\hline Ignorado & 72 & 4,4 & 120 & 7,9 & 57 & 3,4 & 249 & 5,2 \\
\hline Total & 1635 & 100 & 1528 & 100 & 1657 & 100 & 4820 & 100 \\
\hline \multicolumn{9}{|l|}{ Peso (g) ao nascer } \\
\hline$<2500$ & 192 & 11,7 & 185 & 12,1 & 186 & 11,2 & 563 & 11,7 \\
\hline $2500-2999$ & 426 & 26,1 & 424 & 27,8 & 442 & 26,7 & 1292 & 26,8 \\
\hline$\geq 3000$ & 1017 & 62,2 & 919 & 60,1 & 1029 & 62,1 & 2965 & 61,5 \\
\hline Total & 1635 & 100 & 1528 & 100 & 1657 & 100 & 4820 & 100 \\
\hline \multicolumn{9}{|l|}{ Tipos de parto } \\
\hline Vaginal & 881 & 53,9 & 862 & 56,4 & 1010 & 61 & 2753 & 57,1 \\
\hline Cesariana & 727 & 44,5 & 640 & 41,9 & 647 & 39 & 2014 & 41,8 \\
\hline Ignorado & 27 & 1,6 & 26 & 1,7 & - & - & 53 & 1,1 \\
\hline Total & 1635 & 100 & 1528 & 100 & 1657 & 100 & 4820 & 100 \\
\hline
\end{tabular}

Na comparação das adolescentes com mulheres adultas, segundo classificação do MS para idade reprodutiva (10-49 anos), observou-se que as adolescentes apresentam maior chance de terem filhos prematuros do que as mães $>20$ anos $(\mathrm{OR}=1,09)$ (tabela 2). Entretanto, importante ressaltar a considerável quantidade de dados ignorados em relação à IG dos nascidos vivos de mães adolescentes (tabela 1), o que pode estar associado com a coleta e preenchimento das Declarações de Nascidos Vivos (DNV) insatisfatória, demonstrando insuficiência de profissionais qualificados (OLIVEIRA, 2015). 
Tabela 2. Idade materna segundo características da gestação com valores de odds ratio bruto. SINASC, 2012-2014. Feira de Santana - BA.

\begin{tabular}{lcccc}
\hline & \multicolumn{2}{c}{ Duração da gestação } & & \\
Idade materna & $<\mathbf{3 7}$ semanas & $\mathbf{3 7}$ semanas & OR & IC 95\% \\
\hline $10-19$ anos & 252 & 2018 & 1,09 & $0,94-1,25$ \\
$20-49$ anos & 1240 & 10806 & -- & -- \\
TOTAL & 1492 & 12824 & -- & -- \\
\hline
\end{tabular}

$\mathrm{OR}=$ Odds ratio; $\mathrm{IC}=$ Intervalo de confiança $95 \%$

\section{CONSIDERAÇÕES FINAIS}

Conclui-se que há necessidade de melhorias no acompanhamento das gestantes adolescente, pois o pré-natal é a maneira mais adequada de reduzir as taxas de prematuridade, possibilitando melhorias na qualidade de vida da mãe e da criança. Estratégias voltadas para as populações menos favorecidas são essenciais para aumentar o acesso das adolescentes aos serviços de saúde proporcionando um cuidado adequado. Somado a isso, é necessário também qualificação para as equipes multiprofissionais, durante as consultas, o profissional deve permitir que a gestante expresse suas preocupações e angústias, garantindo para elas um prénatal qualificado, demonstrando a importância da continuidade da assistência por profissionais habilitados.

\section{REFERÊNCIAS}

BRASIL. MINISTÉRIO DA SAÚDE. Política nacional de atenção integral à saúde da mulher: princípios e diretrizes, Brasília - DF, 2004.

BRASIL. Ministério da Saúde. Cadernos de Atenção Básica, n 32: Atenção ao pré-natal de baixo risco- - 1. ed. rev. 318 p., Brasília, 2013.

BRASIL. Secretaria de Estado da Saúde. Manual de Neonatologia. Agosto/2015.

FERNANDES, M. M. S. M. et al. Risk factors associated with teenage pregnancy/ Fatores de riscos associados à gravidez na adolescência. Revista de Enfermagem da UFPI, v. 6, n. 3, p. 53-58, 2017.

GAMA, S. G. N. et al. Fatores associados à cesariana entre primíparas adolescentes no Brasil, 2011-2012. Cadernos de Saúde Pública, v. 30, p. S117-S127, 2014.

OLIVEIRA, M. M. et al. Avaliação do Sistema de Informações sobre nascidos vivos. Brasil, 2006 a 2010. Epidemiologia e Serviços de Saúde, v. 24, p. 629-640, 2015.

PONTES, A. M.; et al. As repercussões do aleitamento materno exclusivo em crianças com baixo peso ao nascer. Saúde em Debate, v. 37, p. 354-361, 2013.

RODRIGUES, S. M. S; ALMEIDA, S. S; RAMOS, E. M. L. S. Suporte familiar e transtornos mentais comuns em adolescentes grávidas. Psicologia Argumento, v. 29, n. 64, 2017.

SANTOS, N. L. A. C.; et al. Gravidez na adolescência: análise de fatores de risco para baixo peso, prematuridade e cesariana. Ciênc. saúde coletiva, v.19, n.3, p.719-726, 2014. 